Vol. 4, No. 1, 2018

\author{
Andrij Andrukhiv ${ }^{1}$, Bohdan Sokil ${ }^{2}$, Mariia Sokil $^{3}$ \\ ${ }^{1}$ Scientific Library, Lviv Polytechnic National University, 1, Profesorska Street, Lviv, Ukraine, \\ E-mail: andriy.i.andrukhiv@lpnu.ua \\ ${ }^{2}$ Department of Engineering Mechanics, Hetman Petro Sahaidachny National Army Academy, \\ 32, Heroes of Maidan Str., Lviv, Ukraine, E-mail: Bogdan.I.Sokil@lpnu.ua \\ ${ }^{3}$ Department of Transport Technologies, Lviv Polytechnic National University, 12, S. Bandera Str., \\ Lviv, Ukraine, E-mail: Mariia.B.Sokil@lpnu.ua
}

\title{
RESONANT PHENOMENA OF ELASTIC BODIES THAT PERFORM BENDING AND TORSION VIBRATIONS
}

Received: May 05, 2018 / Revised: June 23, 2018 / Accepted: June 26, 2018

(C) Andrukhiv A., Sokil B., Sokil M., 2018

\begin{abstract}
The method of study of the influence of torsional oscillations of one-dimensional models of nonlinear elastic bodies, along which moves with a constant velocity continuous flow of inelastic homogeneous medium, into bending, is developed. It is believed that information on torsional oscillations is known from empirical studies. Based on the latter, the refined model of the dynamics of the process of the investigated object is constructed. The latter is a boundary value problem for nonlinear nonautonomous differential equations with partial derivatives. The imposed restrictions on power factors and the main parameters of torsional oscillations allow for the analytical study of the dynamics of the process to use the basic ideas of the asymptotic integration of equations with partial derivatives. With their help, we obtain a two-parameter set of solutions that describe the determinant parameters of bending vibrations of an elastic body. It is established that for the considered elastic body there can be resonance oscillations, which are caused not only by external factors, but also by internal - torsional oscillations. Regarding the law of the change in the basic parameters of the dynamics of the bending motion of an elastic body, its rotation around the vertical axis reduces the frequency of its own flexural oscillations of the body, and even small torsional oscillations cause an additional periodic action on the transverse. In connection with the above bending vibrations of the elastic body, which performs complex oscillations (torsion and bending), resonances are possible both at the frequency of the external periodic perturbation and at the frequencies of the torsional oscillations (internal resonances).

The amplitude of the transition through the resonance: a) at the basic frequency of external perturbation takes less value for elastic bodies of greater flexural rigidity and for higher values of the relative motion of the medium; b) at the frequency of torsional oscillations for larger values of the angular velocity takes more importance; c) with "fast" transition through resonance at the frequency of external or internal perturbation is less than with "slow". The obtained results can serve as the basis for the choice of operating parameters of elastic elements of machines that carry out complex oscillations.
\end{abstract}

Keywords: nonlinear-elastic body; asymptotic methods; amplitude; resonance; complex fluctuations.

\section{Introduction}

The determination of the spectrum of eigenfrequencies, and thus, the conditions of the existence of their external and internal resonances, and the peculiarities of their passage, is an important problem in the study of various kinds of complex mechanical systems with lumped masses and distributed parameters. Such problems were partly considered in $[1,2]$ for linear or quasilinear analogues with lumped masses, but for nonlinear systems with distributed parameters the study did not find proper development due to the 
existing complexity of constructing solutions of the corresponding mathematical analogues of their motion [3, 4]. This primarily concerns the effects of torsional oscillations on bending or longitudinal one-dimensional elastic bodies, or vice versa. The indicated problems are considerably complicated for such important classes of mechanical systems with distributed parameters that are characterized by a longitudinal component of the speed of motion [5-8]: the study of the dynamics of one or twodimensional elastic bodies that move with a constant or variable speed of motion and simultaneously carry out bending vibrations and longitudinal oscillations; the effect of torsional oscillations on the bending pipeline or a screw working organ along which a continuous flow of medium moves, etc. Such tasks are the subject of consideration of this work. For a partial solution of this problem in the paper, we make a physically grounded assumption of the existence of necessary information about the laws of changing the basic parameters of some oscillations (for example, twisting) and taking into account the mathematical models indicated by others. In this case, the use of existing analytical methods [9-13] for the construction and research of "refined" solutions, much simpler mathematical models allows us to estimate the influence of a wide range of external and internal factors on the dynamics of these systems. This approach, as shown below, is one of the most affordable in solving many important engineering tasks.

\section{Materials and Methods}

It is known [11] that the mathematical model of bending oscillations of an elastic rectilinear body that rotates along a fixed axis with a constant angular velocity $\Omega$, provided that the continuous flow of medium (liquid, friable or viscous medium) along it is moving along with a relative linear velocity $V$ is the boundary problems for a system of differential equations:

$$
\begin{aligned}
& L_{1}(u, w)=\left(\rho_{1}+\rho_{2}\right) \frac{\partial^{2} u}{\partial t^{2}}+2 \rho_{2} V \frac{\partial^{2} u}{\partial t \partial z}-2\left(\rho_{1}+\rho_{2}\right) \Omega \frac{\partial w}{\partial t}-\left(S-\rho_{2} V^{2}\right) \frac{\partial^{2} u}{\partial z^{2}}- \\
& -2\left(\rho_{1}+\rho_{2}\right) I \Omega \frac{\partial^{3} w}{\partial t \partial x^{2}}+E I \frac{\partial^{4} u}{\partial z^{4}}-\left(\rho_{1}+\rho_{2}\right) \Omega^{2} u=\varepsilon f_{1}\left(u, w, \frac{\partial u}{\partial t}, \ldots, \frac{\partial^{3} w}{\partial z^{3}}, \gamma\right) \\
& L_{2}(u, w)=\left(\rho_{1}+\rho_{2}\right) \frac{\partial^{2} w}{\partial t^{2}}+2 \rho_{2} V \frac{\partial^{2} w}{\partial t \partial z}+2\left(\rho_{1}+\rho_{2}\right) \Omega \frac{\partial u}{\partial t}-\left(S-\rho_{2} V^{2}\right) \frac{\partial^{2} w}{\partial z^{2}}+ \\
& +2\left(\rho_{1}+\rho_{2}\right) I \Omega \frac{\partial^{3} u}{\partial t \partial x^{2}}+E I \frac{\partial^{4} w}{\partial z^{4}}-\left(\rho_{1}+\rho_{2}\right) \Omega^{2} w=\varepsilon f_{2}\left(u, w, \frac{\partial u}{\partial t}, \ldots, \frac{\partial^{3} w}{\partial z^{3}}, \gamma\right)
\end{aligned}
$$

in which (1) $u(t, z), w(t, z)$ are the components of the moving vector of a point that coincides with the center of the cross section of an elastic one-dimensional body at an arbitrary time in projections on the axis of a fixed coordinate system $O X Y Z$ (the axis $O Z$ coincides with the geometric position of the centers of the body sections in its undeformed rectilinear state), $\rho_{1}, \rho_{2}$ are respectively the unit mass the length of the body and the moving environment, $S$ is the tensile force, $\Omega$ is the angular velocity of the body around the axis, $O Z, E I$ is its stiffness to the bend, and $f_{1}\left(u, w, \frac{\partial u}{\partial t}, \ldots, \frac{\partial^{3} w}{\partial z^{3}}, \gamma\right), f_{2}\left(u, w, \frac{\partial u}{\partial t}, \ldots, \frac{\partial^{3} w}{\partial z^{3}}, \gamma\right)$ are the functions that describe the strength of the resistance, the external periodic perturbations with the frequency $\mu$, and other forces, the maximum value of which is significantly smaller than the maximum values of the right-hand side plots, as indicated by the small parameter $\varepsilon,\left(\gamma=\mu t+\theta_{0}, \theta_{0}\right.$ is the initial phase of the indicated perturbation). In addition, their content implies that the specified functions satisfy the condition $f_{1}\left(u, w, \frac{\partial u}{\partial t}, \ldots, \frac{\partial^{3} w}{\partial z^{3}}, \gamma\right)=f_{2}\left(w, u, \frac{\partial w}{\partial t}, \ldots, \frac{\partial^{3} u}{\partial z^{3}}, \gamma\right)$. If, however, the elastic body carries 
torsional oscillations around the axis in accordance with the law, then the system of equations (1) is transformed into a kind

$$
\begin{aligned}
& \mathscr{L}_{1}(u, w)=\left(\rho_{1}+\rho_{2}\right) \frac{\partial^{2} u}{\partial t^{2}}+2 \rho_{2} V \frac{\partial^{2} u}{\partial t \partial z}-2\left(\rho_{1}+\rho_{2}\right)\left(\Omega+\frac{\partial \vartheta(x, t)}{\partial t}\right) \frac{\partial w}{\partial t}-\left(S-\rho_{2} V^{2}\right) \frac{\partial^{2} u}{\partial z^{2}}- \\
& -2\left(\rho_{1}+\rho_{2}\right) I\left(\Omega+\frac{\partial \vartheta(x, t)}{\partial t}\right) \frac{\partial^{3} w}{\partial t \partial x^{2}}+E I \frac{\partial^{4} u}{\partial z^{4}}-\left(\rho_{1}+\rho_{2}\right)\left(\Omega+\frac{\partial \vartheta(x, t)}{\partial t}\right)^{2} u- \\
& -\left(\rho_{1}+\rho_{2}\right) \frac{\partial^{2} \vartheta(x, t)}{\partial t^{2}} w=\varepsilon f_{1}\left(u, w, \frac{\partial u}{\partial t}, \ldots, \frac{\partial^{3} w}{\partial z^{3}}, \gamma\right), \\
& \check{L}_{2}^{\circ}(u, w)=\left(\rho_{1}+\rho_{2}\right) \frac{\partial^{2} w}{\partial t^{2}}+2 \rho_{2} V \frac{\partial^{2} w}{\partial t \partial z}+2\left(\rho_{1}+\rho_{2}\right)\left(\Omega+\frac{\partial \vartheta(x, t)}{\partial t}\right) \frac{\partial u}{\partial t}-\left(S-\rho_{2} V^{2}\right) \frac{\partial^{2} w}{\partial z^{2}}+ \\
& +2\left(\rho_{1}+\rho_{2}\right) I\left(\Omega+\frac{\partial \vartheta(x, t)}{\partial t}\right) \frac{\partial^{3} u}{\partial t \partial x^{2}}+E I \frac{\partial^{4} w}{\partial z^{4}}-\left(\rho_{1}+\rho_{2}\right)\left(\Omega+\frac{\partial \vartheta(x, t)}{\partial t}\right)^{2} w+ \\
& +\left(\rho_{1}+\rho_{2}\right) \frac{\partial^{2} \vartheta(x, t)}{\partial t^{2}} u=\varepsilon f_{2}\left(u, w, \frac{\partial u}{\partial t}, \ldots, \frac{\partial^{3} w}{\partial z^{3}}, \gamma\right) .
\end{aligned}
$$

For the differential equations (2) boundary conditions of the form are considered

$$
\left.w(t, z)\right|_{z=j}=\left.\frac{\partial^{2} w}{\partial z^{2}}\right|_{z=j}=0, j=0, l,\left.\quad u(t, z)\right|_{z=j}=\left.\frac{\partial^{2} u}{\partial z^{2}}\right|_{z=j}=0,
$$

which correspond to the rotation of the elastic body in ideal bearings, the distance between which is equal to $l$.

\section{Solving Procedure}

The task is to comprehensively determine the influence of the main external and internal factors, the motion of the medium along the body, the angular velocity of its rotation around the vertical axis and the torsional oscillations on the bending vibrations, provided that the torsional oscillations are described by a known continuous function $\vartheta(t)$. When solving a given problem it is considered that: a) the medium that moves along the body does not change its stiffness in bending and torsion; $b$ ) torsional oscillations, the nature of which is not considered, is of small amplitude and is described by the dependence [15]

$$
\vartheta(x, t)=a \sin \frac{k \pi}{l} z \cos \vartheta_{k}, \vartheta_{k}=\left(\omega_{\vartheta k} t+\vartheta_{0}\right)
$$

where $a$ is their amplitude, $\omega_{\vartheta k}=\frac{k \pi}{l} \sqrt{\frac{G J_{0}}{I_{0}}}, \vartheta_{0}$ is the frequency, $k=1,2, \ldots, I_{0}$ is the initial phase, and is the torque of inertia relative to the neutral axis of the elastic body, together with the medium, $J_{0}$ is its equatorial moment of inertia, and $G$ is the shear modulus.

The constraints on force factors and torsional oscillations of the considered system, the continuous flow of the medium is an elastic body, allow for the construction of the solution of the boundary value problem (2), (3) under the condition (4) to use the basic ideas of asymptotic methods [1, 9, 10, 13 ]. Accodingly to them, first of all, let's look at their undisturbed analogues, that is, at the equation

$$
L_{1}(u, w)=0, L_{2}(u, w)=0
$$

under homogeneous boundary conditions (3). The principle of one-frequency oscillation for the nonlinear systems with distributed parameters and the basic idea of the D'Almer's method [16], allows us to interpret it as an overlay of the direct and reflected waves of the same frequencies, that is,

$$
\begin{gathered}
u(t, z)=b(\cos (\kappa z+\omega t+\psi)-\cos (\kappa z-\omega t-\varphi)), \\
w(t, z)=b(\sin (\kappa z+\omega t+\psi)+\sin (\kappa z-\omega t-\varphi)),
\end{gathered}
$$


where $b$ is the amplitude of these waves; $\phi, \psi$ are their initial phases; $\kappa, \omega$ are the wave number and frequency, respectively. From the undisturbed differential equations (5), it follows that the parameters are bound by the dispersion relations

$$
\begin{gathered}
\left(\rho_{1}+\rho_{2}\right) \omega^{2}+2\left(\rho_{1}+\rho_{2}\right) \Omega \omega-\left(S-\rho_{2} V^{2}\right) \kappa^{2}-2\left(\rho_{1}+\rho_{2}\right) I \Omega \kappa^{2} \omega- \\
-E I \kappa^{4}+\left(\rho_{1}+\rho_{2}\right) \Omega^{2}=0, \\
\left(\rho_{1}+\rho_{2}\right) \omega^{2}-2\left(\rho_{1}+\rho_{2}\right) \Omega \omega-\left(S-\rho_{2} V^{2}\right) \kappa^{2}+2\left(\rho_{1}+\rho_{2}\right) I \Omega \kappa^{2} \omega- \\
-E I \kappa^{4}+\left(\rho_{1}+\rho_{2}\right) \Omega^{2}=0 .
\end{gathered}
$$

We note that somewhat simpler descriptions of wave processes for quasilinear and nonlinear mathematical models of "long" systems with distributed parameters (for which boundary conditions are not considered) are given, for example, in $[9,10,12]$. For the considered case, that is, from the boundary conditions (3) and the relations (6) and (7) follows: $\phi=\psi, \kappa_{s}=\frac{s \pi}{l}, s=1,2, \ldots$, and the frequency of the dynamic process of undisturbed motion is determined by the system parameters dependence

$$
\omega_{s}=\Omega\left(I \kappa_{s}^{2}-1\right)+\kappa_{s} \sqrt{\Omega^{2} I\left(\kappa_{s}^{2} I-2\right)+\frac{\left(S-\rho_{2} V^{2}\right)+E I \kappa_{s}^{2}}{\rho_{1}+\rho_{2}}} .
$$

Thus, the undisturbed motion of the system in one of the forms of "dynamic equilibrium" is described by the dependence

$$
\begin{aligned}
& u_{s}(t, z)=b_{s}\left(\cos \left(\frac{s \pi}{l} z+\omega_{s} t+\phi_{s}\right)-\cos \left(\frac{s \pi}{l} z-\omega_{s} t-\phi_{s}\right)\right) \\
& w_{s}(t, z)=b_{s}\left(\sin \left(\frac{s \pi}{l} z+\omega_{s} t+\phi_{s}\right)+\sin \left(\frac{s \pi}{l} z-\omega_{s} t-\phi_{s}\right)\right),
\end{aligned}
$$

and the parameters $b_{s}, \phi_{s}$ for the given case are constant and are determined by the initial conditions. In the case of a more complex perturbation motion, these parameters will be determined additionally by the right-hand sides of the differential equations (2) and the law of torsional oscillations of the system. We find the main relations that determine them based on the distribution of the basic ideas of the methods of Bubnov-Galerkin and Van der Pol [1, 17]. In accordance with them, first of all we find

$$
\begin{aligned}
& \frac{\partial u_{s}(t, z)}{\partial t}=-b_{s}\left(\omega_{s}+\frac{d \phi_{s}}{d t}\right)\left(\sin \left(\kappa_{s} z+\psi_{s}\right)+\sin \left(\kappa_{s} z-\psi_{s}\right)\right)+\frac{d b_{s}}{d t}\left(\cos \left(\kappa z+\psi_{s}\right)-\cos \left(\kappa z-\psi_{s}\right)\right), \\
& \frac{\partial w_{s}(t, z)}{\partial t}=b_{s}\left(\omega_{s}+\frac{d \phi}{d t}\right)\left(\cos \left(\kappa_{s} z+\psi_{s}\right)-\cos \left(\kappa_{s} z-\psi_{s}\right)\right)+\frac{d b_{s}}{d t}\left(\sin \left(\kappa_{s} z+\psi_{s}\right)+\sin \left(\kappa_{s} z-\psi_{s}\right)\right) .
\end{aligned}
$$

The basic idea of the Van der Pol's method for the system with small nonlinearity is

$$
\begin{gathered}
-b_{s} \frac{d \phi_{s}}{d t}\left(\sin \left(\kappa_{s} z+\psi_{s}\right)+\sin \left(\kappa_{s} z-\psi_{s}\right)\right)+\frac{d b_{s}}{d t}\left(\cos \left(\kappa z+\psi_{s}\right)-\cos \left(\kappa z-\psi_{s}\right)\right)=0, \\
b_{s} \frac{d \phi}{d t}\left(\cos \left(\kappa_{s} z+\psi_{s}\right)-\cos \left(\kappa_{s} z-\psi_{s}\right)+\frac{d b_{s}}{d t}\left(\sin \left(\kappa_{s} z+\psi_{s}\right)+\sin \left(\kappa_{s} z-\psi_{s}\right)\right)=0 .\right.
\end{gathered}
$$

Differentiating (10) with respect to tome variable and taking into account (11), we conclude

$$
\begin{gathered}
\frac{\partial^{2} u_{s}(t, z)}{\partial t^{2}}=-\frac{d b_{s}}{d t} \omega_{s}\left(\sin \left(\kappa_{s} z+\psi_{s}\right)+\sin \left(\kappa_{s} z-\psi_{s}\right)\right)+ \\
+b_{s} \omega_{s}\left(\frac{d \phi_{s}}{d t}+\omega_{s}\right)\left(\cos \left(\kappa_{s} z+\psi_{s}\right)-\cos \left(\kappa_{s} z-\psi_{s}\right)\right),
\end{gathered}
$$




$$
\begin{gathered}
\frac{\partial^{2} w(t, z)}{\partial t^{2}}=\frac{d b_{s}}{d t} \omega_{s}\left(\cos \left(\kappa_{s} z+\psi_{s}\right)-\cos \left(\kappa_{s} z-\psi_{s}\right)\right)- \\
-b_{s} \omega_{s}\left(\frac{d \phi_{s}}{d t}+\omega_{s}\right)\left(\sin \left(\kappa_{s} z+\psi_{s}\right)+\sin \left(\kappa_{s} z-\psi_{s}\right)\right) .
\end{gathered}
$$

If we substitute in the base equation (2) in the place of unknown functions $u(z, t), w(z, t)$ and their derivative dependences accordingly to (4), (9) - (12), we obtain a system of differential equations that binds unknown parameters $b_{s}$ and $\phi_{s}$

$$
\begin{gathered}
\frac{d b_{s}}{d t} \omega_{s} \sin \kappa_{s} z \cos \psi_{s}-b_{s} \omega_{s} \frac{d \varphi_{s}}{d t} \sin \kappa_{s} z \sin \psi_{s}=f\left(\rho b_{1}, z, \psi_{1}, \gamma\right)+2 \frac{\rho_{2} b_{s} V \kappa_{s}}{\rho_{1}+\rho_{2}} \cos \kappa_{s} z \cos \psi_{s}+ \\
+2 a b_{s} \omega_{s} \omega_{\vartheta k} \sin \kappa_{s} z \sin \psi_{s} \cdot\left[\left(1-I \kappa_{s}^{2}+\frac{\Omega}{\omega_{s}}\right) \sin \vartheta_{k} \sin \psi_{s}-\frac{\omega_{\vartheta k}}{\omega_{s}} \cos \vartheta_{k} \cos \psi_{s}\right], \\
\frac{d b_{s}}{d t} \omega_{s} \sin \kappa_{s} z \sin \psi_{s}+b_{s} \omega_{s} \frac{d \varphi_{s}}{d t} \sin \kappa_{s} z \cos \psi_{s}=0,
\end{gathered}
$$

where and a well-known function that corresponds to the value of the right-hand side of the differential equations (2), provided that they are in accordance with (6), (10) - (12). From the system of differential equations (13) we find

$$
\begin{gathered}
\frac{d b_{s}}{d t}=\frac{\varepsilon}{2 \omega_{s}} f^{q}\left(b_{1}, z, \psi_{1}, \gamma\right) \sin \kappa_{s} z \cos \psi_{s}+\frac{\rho_{2} b_{s} V \kappa_{s}}{\rho_{1}+\rho_{2}} \omega_{s} \sin 2 \kappa_{s} z \cos ^{2} \psi_{s}+a b_{s} \omega_{s} \omega_{\vartheta k} \sin ^{2} \kappa_{s} z \times \\
\times\left[\left(1-I \kappa_{s}^{2}+\frac{\Omega}{\omega_{s}}\right) \sin 2 \psi_{s} \sin \left(\omega_{\vartheta k} t+\vartheta_{0}\right)-2 \frac{\omega_{\vartheta k}}{\omega_{s}} \cos \left(\omega_{\vartheta k} t+\vartheta_{0}\right) \cos ^{2} \psi_{s}\right], \\
\frac{d \psi_{s}}{d t}=\omega_{s+} \frac{\varepsilon}{2 b_{s} \omega_{s}} f /\left(b_{1}, z, \psi_{1}, \gamma\right) \sin \kappa_{s} z \sin \psi_{s}-\frac{\rho_{2} b_{s} V \kappa_{s}}{\rho_{1}+\rho_{2}} \omega_{s} \sin 2 \kappa_{s} z \sin 2 \psi_{s}- \\
-2 a b_{s} \omega_{s} \omega_{\vartheta k} \sin ^{2} \kappa_{s} z \cdot\left[\left(1-I \kappa_{s}^{2}+\frac{\Omega}{\omega_{s}}\right) \sin ^{2} \psi_{s} \sin \left(\omega_{\vartheta k} t+\vartheta_{0}\right)-\frac{\omega_{\vartheta k}}{2 \omega_{s}} \cos \left(\omega_{\vartheta k} t+\vartheta_{0}\right) \sin 2 \psi_{s}\right] .
\end{gathered}
$$

The resulting system of differential equations (14) allows us to assert that nonresonance and resonance oscillations may occur in the corresponding mechanical system. As for the latter, they exist under certain conditions at the frequency of external periodic perturbation and at frequencies of multiple frequencies of torsional oscillations. We consider initially a simpler, nonresonant, case of bending vibrations of the system under consideration. For the first approximation, the basic parameters of bending vibrations of an elastic body in the form close to the first form of "dynamic equilibrium", as follows from the differential equations (14), are described by dependencies

$$
\begin{aligned}
& \frac{d b_{1}}{d t}=\frac{-\varepsilon}{8 \omega_{1} \pi^{2} l} \int_{0}^{l} \int_{0}^{2 \pi} \int_{0}^{2 \pi} f /\left(o b_{1}, z, \psi_{1}, \gamma\right) \sin \frac{\pi}{l} z \cos \psi_{1} d z d \psi_{1} d \gamma, \\
& \frac{d \psi_{1}}{d t}=\omega_{1}-\frac{\varepsilon}{8 \omega_{1} \pi^{2} b_{1} l} \int_{0}^{l} \int_{0}^{2 \pi} \int_{0}^{2 \pi} f /\left(b_{1}, z, \psi_{1}, \gamma\right) \sin \frac{\pi}{l} z \sin \psi_{1} d z d \psi_{1} d \gamma .
\end{aligned}
$$

\section{Results and Discussion}

Thus, in the first approximation for the nonresonant oscillations of the system under consideration, the basic parameters of the bending oscillations of the system are not continuous oscillations of the tidal 
oscillations. As to the resonance at the frequency of the external periodic perturbation, the amplitude of the transition through the resonance is described by the dependence

$$
\begin{aligned}
& \frac{d b_{1}}{d t}=\frac{-\varepsilon}{4 \omega_{1} \pi l} \int_{0}^{l} \int_{0}^{2 \pi} f /\left(b_{1}, z, \gamma+\varphi_{1}, \gamma\right) \sin \frac{\pi}{l} z \cos \left(\gamma+\varphi_{1}\right) d z d \gamma \\
& \frac{d \varphi_{1}}{d t}=\omega_{1}-\mu-\frac{\varepsilon}{8 \omega_{1} \pi^{2} b_{1} l} \int_{0}^{l} \int_{0}^{2 \pi} q /\left(b_{1}, z, \gamma+\varphi_{1}, \gamma\right) \sin \frac{\pi}{l} z \sin \left(\gamma+\varphi_{1}\right) d z d \gamma,
\end{aligned}
$$

where $\varphi_{1}$ denotes the phase difference between the self-bending and forced oscillations, i.e. $\varphi_{1}=\psi_{1}-\gamma$.

In Fig. 1, for various values of $f_{1}(\ldots)=\delta\left(u_{t}\right)^{2 r+1}+h \cos \gamma$ the system parameters, the law of variation of the amplitude during the transition of the principal resonance at the frequency of the external periodic perturbation is presented.
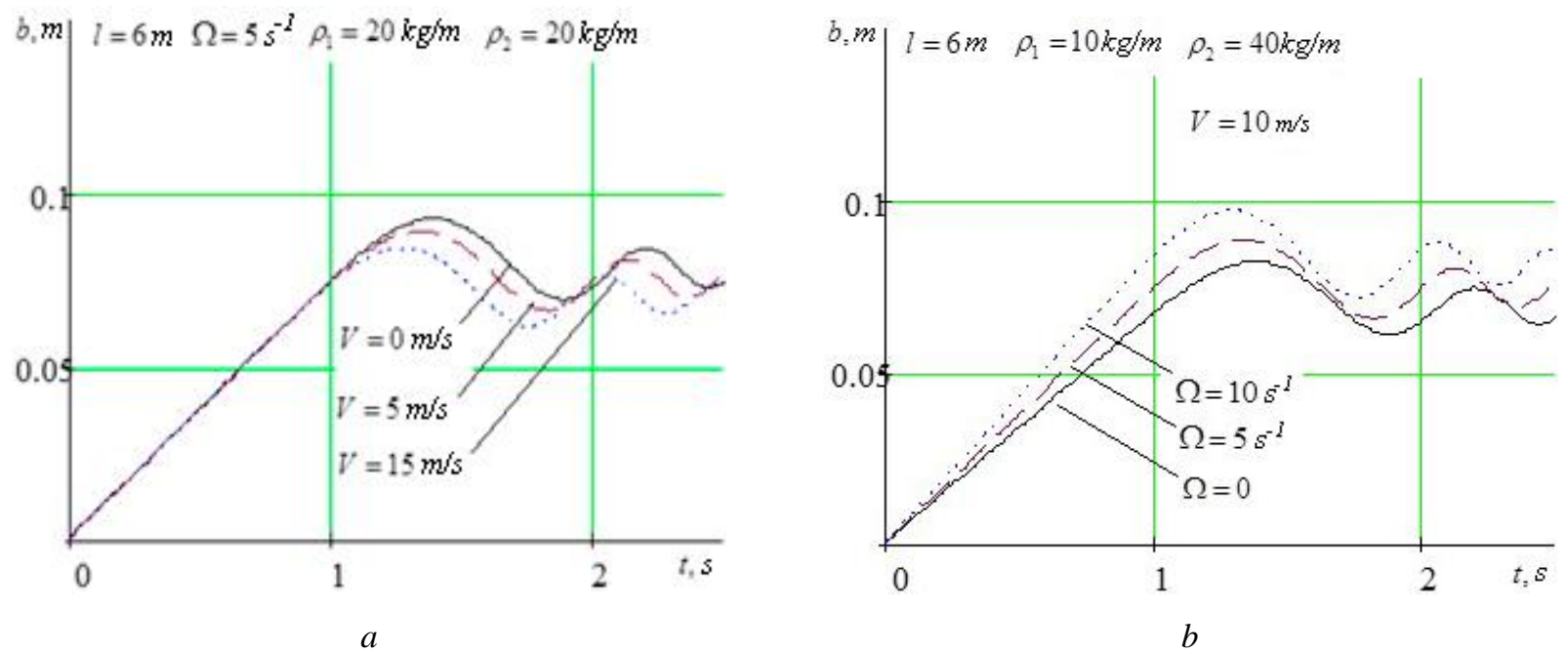

Fig. 1. The law of the change of the amplitude of transverse oscillations during the passage of resonance at the frequency of external periodic perturbation for different values of the angular velocity of the body and the velocities of the medium

The obtained dependences show that for: a) the larger values of the uniform mass of the medium, the amplitude of the transition through the resonance is greater; b) for larger values of the angular velocity of the body's rotation, the actual frequency of bending oscillations is smaller at the same time, the amplitude of the transition through the resonance is smaller; c) at a higher speed of transition through resonance of the amplitude is less.

A more complex case is the resonance oscillations, which are due to torsional oscillations. Let us consider them in more detail for the case when the forms of transverse and torsional oscillations are close in terms of the frequency $\omega_{s}=\omega_{\vartheta k}$. In this case, in the terms of the right-hand side of the general relations (14), which determine the influence on the dynamics of the system of torsional oscillations or the motion of the medium, the expressions $\cos ^{2} \psi_{s}$ and $\sin ^{2} \psi_{s}$ we replace on $\frac{1}{2}\left(1+\cos 2 \psi_{s}\right)$ and $\frac{1}{2}\left(1-\cos 2 \psi_{s}\right)$, so the system of differential equations (14), after the averaging over the linear variable and the phase of external perturbation transformed into a kind

$$
\begin{aligned}
& \frac{d b_{1}}{d t}=\frac{-\bar{\varepsilon}}{4 \omega_{s} \pi l} \int_{0}^{l} \int_{0}^{2 \pi} f\left(b_{1}, z, \psi_{s}, \gamma\right) \sin \kappa_{s} z \cos \psi_{s} d z d \gamma+a b_{s} \omega_{s} \omega_{\vartheta k} \delta_{s}^{k} \times \\
& \times\left[\left(1-I \kappa_{s}^{2}+\frac{\Omega}{\omega_{s}}\right) \sin 2\left(\omega_{s} t+\varphi_{s}\right) \sin \left(\omega_{\vartheta k} t+\vartheta_{0}\right)-\frac{\omega_{\vartheta k}}{\omega_{s}}\left(1+\cos \left(\omega_{s} t+\varphi_{s}\right)\right) \cos \left(\omega_{\vartheta k} t+\vartheta_{0}\right)\right],
\end{aligned}
$$




$$
\begin{aligned}
& \frac{d \psi_{s}}{d t}=\omega_{s}+\frac{-\bar{\varepsilon}}{4 \omega_{s} b_{s} \pi l} \int_{0}^{l} \int_{0}^{2 \pi} g /\left(b_{1}, z, \psi_{s}, \gamma\right) \sin \kappa_{s} z \sin \psi_{s} d z d \gamma+a \omega_{s} \omega_{\vartheta k} \delta_{s}^{k} \times \\
& \times\left[\left(1-I \kappa_{s}^{2}+\frac{\Omega}{\omega_{s}}\right)\left(1-\cos 2\left(\omega_{s} t+\varphi_{s}\right)\right) \sin \left(\omega_{\vartheta k} t+\vartheta_{0}\right)-\frac{\omega_{\vartheta k}}{2 \omega_{s}} \sin 2\left(\omega_{s} t+\varphi_{s}\right) \cos \left(\omega_{\vartheta k} t+\vartheta_{0}\right)\right],
\end{aligned}
$$

where $\delta_{s}^{k}=\frac{1}{l} \int_{0}^{l} \sin \frac{k \pi}{l} z \sin ^{2} \kappa_{s} z d z$

The relations (17) show that, in the case of the fulfilment of the conditions $2 \omega_{s} \approx \omega_{\vartheta k}$ in the system, there will exist resonance transverse oscillations that are due to torsional oscillations. To describe them, in the same way as for resonant oscillations in the frequency of the external periodic perturbation, introducing into the equation (17) the phase difference $\varphi_{2}=2 \psi_{s}-\vartheta_{k}\left(\varphi_{2}+\vartheta_{k}=2 \psi_{s}\right)$ from (17) we obtain

$$
\begin{gathered}
\frac{d b_{1}}{d t}=\frac{-\bar{\varepsilon}}{4 \omega_{s} \pi l} \int_{0}^{l} \int_{0}^{2 \pi} f\left(b_{1}, z, \psi_{s}, \gamma\right) \sin \kappa_{s} z \cos \psi_{s} d z d \gamma+a b_{s} \omega_{s} \omega_{\vartheta k} \delta_{s}^{k}\left(1-I \kappa_{s}^{2}+\frac{\Omega}{\omega_{1}}-\omega_{\vartheta 1}\right) \cos \varphi_{2} \\
\left.\frac{d \varphi_{2}}{d t}=2 \omega_{s}-\omega_{\vartheta /}+\frac{-\bar{\varepsilon}}{4 \omega_{s} b_{s} \pi l} \int_{0}^{l} \int_{0}^{2 \pi} f / \rho b_{1}, z, \psi_{s}, \gamma\right) \sin \kappa_{s} z \sin \psi_{s} d z d \gamma+ \\
+a \omega_{s} \omega_{\vartheta k} \delta_{1}^{k}\left(1-I \kappa_{1}^{2}+\frac{\Omega}{\omega_{1}}-\omega_{\vartheta k}\right) \sin \varphi_{2} .
\end{gathered}
$$

If the function $f_{1}\left(u, w, \frac{\partial u}{\partial t}, \ldots, \frac{\partial^{3} w}{\partial z^{3}}, \gamma\right)$ takes into account only the strength of the resistance and the external periodic perturbation, that is, expressed by the relation (16), then the transverse resonance oscillations are due to the torsional oscillations in the case are described by the differential equations

$$
\begin{aligned}
& \frac{d b_{1}}{d t}=-\varepsilon \omega^{2 r} b_{1}^{2 r}+\frac{4}{3} a b_{1} \omega_{\vartheta 1}\left(1-I \kappa_{1}^{2}+\frac{\Omega}{\omega_{1}}-\frac{\omega_{\vartheta 1}}{\omega_{1}}\right) \cos \varphi_{2}, \\
& \frac{d \varphi_{2}}{d t}=2 \omega_{1}-\omega_{\vartheta 1}+\frac{4}{3} a \omega_{\vartheta 1}\left(1-I \kappa_{1}^{2}+\frac{\Omega}{\omega_{1}}-\frac{\omega_{\vartheta 1}}{\omega_{1}}\right) \sin \varphi_{2} .
\end{aligned}
$$

In Fig. 2, for different values of the parameters, the law of the amplitude changes during the transition of drowsiness on the frequency of the external periodic perturbation is presented.
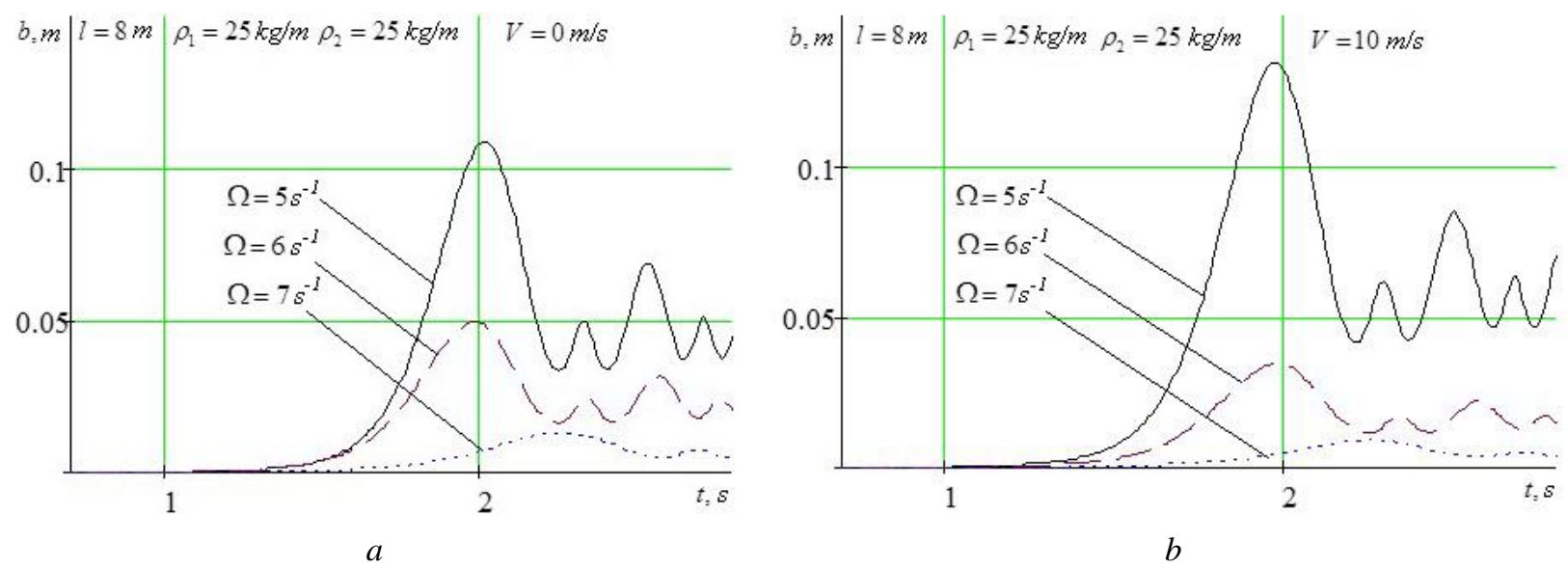

Fig. 2. Time variation of the amplitude of transverse oscillations during passage of internal resonance at different angular velocities of the body 


\section{Conclusions}

On the basis of the developed method of studying the influence of torsional oscillations on transverse elastic one-dimensional bodies, which carry out rotational motion around the vertical axis and motion along its continuous stream of inelastic medium, it is established:

- the angular speed of the screw working body reduces the frequency of its own flexural oscillations of the body, and even small torsional oscillations cause an additional periodic effect on the transverse;

- taking into account the above we conclude that for the bending oscillations of the elastic body, which carries out complex oscillations (twisting and bending) the resonances are possible at frequencies of external periodic perturbation and frequencies of torsional oscillations;

- the amplitude of the transition through the resonance at the fundamental frequency of external perturbation takes less importance for the elastic bodies of the greater stiffness of the nicks;

- the amplitude of the transition through the resonance at the second frequency of torsional oscillations for larger values of the relative motion of the medium takes more significance;

- the amplitude of bending oscillations in the "fast" transition through resonance at the frequency of external or internal disturbance is less than that of "slow".

The obtained results can serve as the basis for choosing the basic technological and operational parameters of the specified type of systems, and their reliability is confirmed by obtaining in the limiting case when the known results relating to flexural oscillations of the elastic body. The main idea of the described method can be generalized to some other classes of mechanical systems.

\section{References}

[1] N. N. Bogoliubov, and Iu. A. Mitropolskii, Asimptoticheskie metody v teorii nelineinykh kolebanii [Asymptotic methods in the theory of nonlinear oscillations]. Moscow, Russia: Nauka Publ., 1974. [in Russian].

[2] D. Ye. Khaustov, "Umovy vnutrishnoho rezonansu viiskovoi husenychnoi tekhniky" ["Conditions of internal resonance of military tracked vehicles"], Systemy ozbroiennia i viiskova tekhnika [Systems of Arms and Military Equipment], no. 1 (45), pp. 73-76, 2016. [in Ukrainian].

[3] P. I. Ohorodnikov, V. M. Svitlytskyi, and V. I. Hohol, "Doslidzhennia zv'iazku mizh pozdovzhnimy i krutylnymy kolyvanniamy burylnoi kolony" ["Investigation of the connection between the longitudinal and torsional oscillations of the drill column"], Naftova haluz Ukrainy [The oil industry of Ukraine], no. 2, pp. 6-9, 2014. [in Ukrainian].

[4] I. A. Lukovskii, A. V. Solodun, and A. N. Timokha, "O vnutrennikh rezonansakh kolebanii zhidkostei v konicheskikh bakakh" ["On the internal resonances of oscillations of liquids in conical tanks"], Prykladna hidromekhanika [Applied hydromechanics], vol. 15, no. 2, pp. 46-52, 2013. [in Russian].

[5] P. Ya. Pukach, I. V. Kuzio, and M. B. Sokil, "Nelineinye izgibnye kolebaniia vrashchaiushchikhsia vokrug nepodvizhnoi osi tel i metodika ikh issledovaniia" ["Non-linear flexural vibrations of revolving bodies around a fixed axis and methods of their study"], Izvestiia vysshikh uchebnykh zavedenii. Gornyi zhurnal [News of the Higher Institutions. Mining Journal], no. 7, pp. 141-149, 2013. [in Russian].

[6] R. M. Rohatynskyi, Naukovo-prykladni osnovy stvorennia hvyntovykh transportno-tekhnolohichnykh mekhanizmiv [Scientific and applied foundations of creation of screw transport and technological mechanisms]. Ternopil, Ukraine: TNTU imeni Ivana Puliuia Publ., 2014. [in Ukrainian].

[7] I. V. Kuzio, Ye. V. Kharchenko, and M. B. Sokil, "Dynamichni protsesy u seredovyshchakh, yaki kharakteryzuiutsia pozdovzhnim rukhom, ta vplyv kraiovykh umov na amplitudu i chastotu yikh kolyvan" ["Dynamic processes in environments characterized by longitudinal motion and the effect of boundary conditions on the amplitude and frequency of their oscillations"], Vibratsii v tekhnitsi i tekhnolohiiakh [Vibrations in technique and technologies], no. 3 (48), pp. 53-56, 2007. [in Ukrainian].

[8] B. M. Hevko, and Yu. F. Pavelchuk, "Modeliuvannia kolyvan mekhanichnoi systemy pidvisnyi soshnyk: teoretychnyi analiz" ["Modeling of fluctuations of the mechanical system of a suspension hole: theoretical analysis"], Innovative solutions in modern science, no. 1 (10), pp. 1-9, 2017. [in Ukrainian].

[9] Y. A. Mitropol'skii, and B. I. Sokil, "On the application of Ateb-functions to the construction of an asymptotic solution of the perturbed nonlinear Klein-Gordon equation”, Ukrainian Mathematical Journal, no. 5 (50), pp. 754-760, 1998. 
[10] B. I. Sokil, "Construction of asymptotic solutions of certain boundary-value problems for the nonautonomous wave equation", Journal of Mathematical Sciences, no. 1 (96), pp. 2878-2882, 1999.

[11] V. I. Huliaiev, and O. I. Borshch, "Spiralni khvyli v zakruchenykh pruzhnykh trubchastykh sterzhniakh, shcho obertaiutsia z vnutrishnim potokom ridyny" ["Spiral waves in twisted elastic tubular rods, rotating with internal fluid flow"], Akustychnyi visnyk [ Acoustic Bulletin], vol. 10, no. 3, pp. 12-18, 2007. [in Ukrainian].

[12] Iu. A. Mitropolskii, "O postroenii asimptoticheskogo resheniia vozmushchennogo uravneniia KleinaGordona" ["On the construction of an asymptotic solution of the perturbed Klein-Gordon equation"], Ukrainskyi matematychnyi zhurnal [Ukrainian Mathematical Journal], vol. 47, no. 9, pp. 1209-1216, 1995. [in Russian].

[13] Iu. A. Mitropolskii, "O postroenii asimptoticheskogo resheniia vozmushchennogo uravneniia KleinaGordona" ["On the construction of an asymptotic solution of the perturbed Brezerton equation"], Ukrainskyi matematychnyi zhurnal [Ukrainian Mathematical Journal], vol. 59, no. 1, pp. 58-71, 1998. [in Russian].

[14] N. S. Pirogova, and P. A. Taranenko, "Raschetno-eksperimentalnyi analiz sobstvennykh i kriticheskikh chastot i form visokooborotnogo rotora mikrogazoturbinnoi ustanovki" ["Calculation-experimental analysis of intrinsic and critical frequencies and forms of a high-speed rotor of a micro-gas turbine unit"], Vestnik IuzhnoUralskogo gosudarstvennogo universiteta. Seriia "Mashinostroenie" [Bulletin of the South Ural State University. Series "Mechanical Engineering Industry"], vol. 15, no. 3, pp. 37-47, 2015. [in Russian].

[15] P. M. Senik, B. I. Sokil, "Ob opredelenii parametrov nelineinoi kolebatelnoi sistemy po amplitudnochastotnoi kharakteristike" ["Non-linear flexural vibrations of revolving bodies around a fixed axis and methods of their study"], Matematicheskie metody i fiziko-mekhanicheskie polia [Mathematical methods and physicomechanical fields], no. 7, pp. 94-99, 1977. [in Russian].

[16] M. B. Sokil, and O. I. Khytriak, "Khvylova teoriia rukhu v doslidzhenni kolyvan hnuchkykh elementiv pryvodu ta transportuvannia z urakhuvanniam yikh pozdovzhnoho rukhu" ["Wave theory of motion in the study of oscillations of flexible elements of the drive and transportation in view of their longitudinal motion"], Viiskovotekhnichnyi zbirnyk [Military Technical Collection], vol. 1, pp. 102-105, 2011. [in Ukrainian].

[17] I. M. Babakov, Teoriia kolebanii [Theory of oscillations]. Moscow, Russia: Nauka Publ., 1965. [in Russian]. 